\title{
Resource Group Assertive Community Treatment (RACT) as a Tool of Empowerment for Clients with Severe Mental Illness: A Meta-Analysis
}

\author{
Tommy Nordén ${ }^{1}$, Ulf Malm ${ }^{2}$ and Torsten Norlander ${ }^{3, *}$ \\ ${ }^{I}$ Evidens Research and Development Center, Göteborg, Sweden \\ ${ }^{2}$ Inst. for Clinical Neuropsychiatry, Sahlgrenska University Hospital, Göteborg, Sweden \\ ${ }^{3}$ Depatment of Clinical Neuroscience, Karolinska Institutet, Solna, Sweden and Evidens Research and Development \\ Center, Göteborg, Sweden
}

\begin{abstract}
The aim of the current meta-analysis was to explore the effectiveness of the method here labeled Resource Group Assertive Community Treatment (RACT) for clients with psychiatric diagnoses as compared to standard care during the period 2001 - 2011. Included in the meta-analysis were 17 studies comprising a total of 2263 clients, 1291 men and 972 women, with a weighted mean age of 45.44 years. The diagnoses of $86 \%$ of the clients were within the psychotic spectrum while $14 \%$ had other psychiatric diagnoses. There were six randomized controlled trials and eleven observational studies. The studies spanned between 12 and 60 months, and 10 of them lasted 24 months. The results indicated a large effect-size for the "grand total measure" (Cohen's $d=0.80$ ). The study comprised three outcome variables: Symptoms, Functioning, and Well-being. With regard to Symptoms, a medium effect for both randomized controlled trials and non-randomized studies was found, whereas Functioning showed large effects for both types of design. Concerning Wellbeing both large and medium effects were evident. The conclusions of the meta-analysis were that the treatment of clients with Resource Group Assertive Community Treatment yields positive effects for clients with psychoses and that the method may be of use for clients within the entire psychiatric spectrum.
\end{abstract}

Keywords: ACT, case manager, empowerment, optimal treatment, RACT, resource group.

\section{INTRODUCTION}

In a systematic review concerned with community mental health team management in severe mental illness [1], standard care was defined as the usual care in the area concerned. In most circumstances this was found to be hospitalbased out-patient care that is characterized by giving clients access to a psychiatrist to get the diagnosis and prescription of medicines, to a social worker to solve practical problems, to a psychologist to get counseling and to the nurse to take blood samples. Coordination of efforts is often inadequate, as the possibilities for clients' families and networks to participate in care. An additional feature of the standard care can often be that there are shortcomings in the monitoring and periodic recordings of clients' condition.

New strategies have evolved from the recognition that standard care is insufficient [1]. There are at present a number of different models for Assertive Community Treatment (ACT) and new experience and research results are constantly reported [2]. ACT models are community based treatment and rehabilitation programs which developed during the 1970 s and 80 s and were intended primarily for individuals with long-term illness [3]. An ACT team is a

*Address correspondence to this author at the Evidens Research and Development Center, Packhusplatsen 2, SE-411 13 Göteborg, Sweden.

Tel: +46 7066211 89; Fax: +46 3171104 42;

E-mail: at.norlander@mailbox.swipnet.se multidisciplinary team that works with intensive clinical case management focused first and foremost on treatment. The treatment is carried out in the client's own immediate surroundings rather than at a clinic or other health-care setting. The original variant was carried out by a purely psychiatric health-care team where all members of the team were capable of working with all of the clients and where all team members could have a case management function [4].

It is the model that originates in the work of the New Zealander Ian Falloon [5, 6] that most radically has taken hold in the field of shared decision making and empowerment of the clients. This integrated and flexible care approach builds upon the evidence and strengths of family management of schizophrenia $[7,8]$ in which the original family unit in the community was expanded by clinical experts to include resource persons from the social network of the client. A crucial difference between the Falloon model and all other ACT models is that the clients themselves and their significant others are part of the ACT team. The concept of Resource Group as the designation of an ACT team where the client and his/her relatives and friends are included emerged in the beginning of the 2000's [9]. The Resource Group method is built on the basic theme that the subjects themselves set the goals for their treatment and have a decisive impact on how the treatment is to be designed [10]. The model has been given a number of different names but since the model continues to be developed with an ever greater emphasis on the central position of the client through the 
participation of the Resource Group the decision was to label the model the Resource group ACT or RACT [11].

In the RACT model [11] it is the client who determines what the overall objectives should be, but the group takes joint decisions about how to achieve them. Resource Group meetings decide on priority and short-term goals for the next three months. The meeting also decides how to proceed in order to evaluate clients' progress. As the meeting is concluded tasks are divided between participants. The psychiatrist may have to alter the medication and the social worker may help with the rent debt. A family member may have to call every morning and have the client get out of bed and a friend can make arrangements with the cinema or some other activity every week. The case manager coordinates the activities and keeps in touch with the resource team members between meetings.

As part of the treatment program the group members are trained [11]. Due to disease and available treatment methods, straightforward information is given based on research results. The case manager and other professionals teach the client and the relatives of some methods that allow them to communicate better, handle stress and solve everyday problems that often feel invincible when you are mentally ill. A very important part in the Resource Group's work is to establish a plan for early intervention schemes if the client seems to approach a relapse into psychosis. A list of signs and an action plan is distributed to those persons in the Resource Group that are appropriate to assist if a crisis is approaching. Efforts are taken that reduces the stress the client is experiencing. If this does not seem to have enough impact alterations are made concerning medications.

The treatment program as a whole has been scientifically researched and field-tested in a number of countries each of which has its own particular system for providing health care and welfare support [12]. Most of the reviews, however, primarily deal with studies conducted during the 1990 's or during the early 2000 's. During this period a picture emerged suggesting that the method "advocated by Falloon has been demonstrated through the scientific literature to be efficient and effective treatment strategies for people experiencing severe mental illness" [13, page 44]. However, a constellation of research studies on Resource Groups comprising the most recent ten-year period is lacking. It is also worth noting that up until now there exists no meta-analysis on Resource group Assertive Community Treatment (RACT).

The RACT model was described in detail by Nordén, et al. [11] in a phenomenological study showing among other results that the Integrated Mental Health Care Workbook, which contains training programs, training protocols, organizational ideas, methodology for follow-up of results, and quality control, is of utmost importance for giving the case manager, jointly with the client, tools for the construction of an effective Resource Group. The fact that the client $\mathrm{him} /$ herself defines his/her own treatment goals, nominates those to be included in the Resource Group and is trained by the case manager to be, if possible, the leader of the Resource Group all seem to be crucial factors in the empowerment of the client. The empowerment of the client, in turn, appears to be the major driving force for successful treatment. The results of the study [11] also indicate that
Resource Groups may be useful both to psychotic clients and clients with other types of diagnoses.

The aim of the current meta-analysis was to explore the effectiveness of the RACT method compared to standard care during the period $2001-2011$. Two hypotheses were put forward: (a) the treatment of clients with the Resource group Assertive Community Treatment (RACT) will yield positive effects for psychotic clients, and (b) the treatment of clients with the Resource-group Assertive Community Treatment (RACT) may be of use to clients within the entire psychiatric spectrum.

\section{MATERIALS AND METHODOLOGY}

\section{Search and Inclusion Criteria}

A search was conducted in the data bases Medline and in Psycinfo $(1980-2011)$ in order to find studies reporting results from psycho-educational interventions using $\mathrm{Re}$ source Groups. In addition to Resource Group and Assertive Community Treatment, other key words were an array of concepts used by the approach emanating from the Optimal Treatment Project [7], e.g. Optimal Treatment (OT), Integrated Treatment (IT), Integrated Care (IC), Integrated Psychiatry (IP), Integrated Mental Health Care (IMHC), Optimal Case Management (OCM), Early Intervention (EI), Behavioral Family Therapy (BFT), as well as Psychoeducational Family Interventions (PFI). The analyses of the results from the search suggested that several quite disparate methods and treatment strategies may have used similar or corresponding concepts and actual resource groups were labeled as resource groups only in the early $2000^{\prime}$ s. In addition, it also became evident that different comparisons were used in the identified studies, ranging from standard care to comparisons among various psycho-educational programs.

Given that the purpose of the current meta-analysis was to explore the effectiveness of the RACT method as compared to standard care, a decision was made to use the following criteria of inclusion: (a) the meta-analysis was to cover the period 2001-2011 in order that contacting relevant authors would be possible, should any lack of clarity exist, (b) results must appear in published manuscripts, reports, or unpublished manuscripts, (c) it must be very clear that the intervention, study, or report was a RACT-program nationally adapted to a specific country's type of health care, social welfare, or welfare in general, (d) the daily implementation was conducted in accordance with the Integrated Mental Health Care Workbook or in nationally adapted translations thereof, (e) relevant control groups and reference groups must be regarded as standard care. In the case of a lack of clarity, regarding e.g. if the methods used clearly followed the instructions outlined in the Integrated Mental Health Care Workbook, or if the control or reference groups needed further definitions, or if it was unclear whether the same data set had been used on several occasions, then the relevant author of the study was contacted. In addition, some ten people within the international network of the Optimal Treatment Project were contacted in order to inquire if they knew of additional published papers emanating from the relevant time period, and if they would also pose the same question to others. Our strategy yielded 17 studies which all met the five criteria of inclusion [10,11, 14-28]. 


\section{Data Extraction and Outcome Variables}

The 17 studies included in the current meta-analysis involved a total of 2263 clients (1291 men and 972 women) and the weighted mean age of the participants was 45.44 years. The diagnoses of $86 \%$ (1955) of the clients were within the psychotic spectrum, while $14 \%$ (308) were found to display the whole range of typical psychiatric diagnoses such as delusional syndrome, addiction syndrome, depression, panic disorder, anxiety disorders, obsessive-compulsive disorder, behavioral disorders, personality disorders, and attentional disorders. Six randomized controlled trials of Resource Group Assertive Community Treatment (RACT) were compared with standard care treatment $[18,20,22,23$, $26,27]$. In addition, there were eleven observational studies where eight compared pre- and post-RACT treatment outcomes $[11,15-17,19,24,25,28]$ and three compared interventions with RACT and the standard care treatment $[10,14$, 21]. Fourteen studies were published in peer-reviewed journals, and the remaining three $[17,19,25]$ were available as reports published in connection with research projects. A few of the studies reported annual results over the course of several years, and in the current meta-analysis the strategy was chosen that given several alternatives, to choose the results obtained at 24 months post-treatment. Thus, for five of the studies the results at 12 months were included [11, 14, $22,23,28]$, for ten of the studies at 24 months $[10,15,17-$ 20, 24-27], for one study at 48 months [16], and for one additional study as late as at 60 months [21].

An examination of the studies showed that the studies contained an array of different measurement instruments. However, it was clear early on that most of them sought to describe one or more domains, e.g. client symptoms, functioning, or the client's sense of well-being. For this reason, it was deemed appropriate to use those three domains as the basis for the outcome variables of the current meta-analysis. Fourteen studies contained various well-known clinical measures of symptoms (e. g., BPRS, CPS, GAF-S, PANSS, PAS). Fifteen studies included well-known clinical measures of functioning (e. g., DAS, GAF-F, PSP) or data by which functioning could be quantified (e. g., hospital use, success rate concerning client's goals for daily chores). Eleven studies included familiar clinical measures of well-being (e. g., Consumer satisfaction, Quality of Life) or other data that in some way could be quantified as well-being (e. g., composite clinical index, friendship, diminished misconduct, reduced stress, healthy attitudes). For studies that used multiple measures for one outcome variable, the effect sizes were averaged into one Cohen's $d$.

\section{Statistical Analyses}

A meta-analysis is a study whose aim is to summarize the results of several independent research studies. In analyzing and testing the relevant studies it is customary to use effect size as the measure of choice [29]. The effect-size, which is the standardized difference between means, is preferred to traditional significance testing [30], as it allows for comparisons between studies with different sample sizes, and even comparisons between different test instruments designed to measure the same underlying phenomenon. There exist a series of measures of the effect size (e.g., Cohen's $d, E t a^{2}$, Glass' delta, Hedges' $g$, odds ratio, Pearson's $r$ ). We chosed
Cohen's $d$ for the current meta-analysis, the most commonly used measure in the psychological and psychiatric literature [31], where most frequently the goal is to describe outcome parameters as continuous measures, as opposed to as categories (e. g., odds ratio). Cohen's $d$ represents the standardized mean difference between either the pre-intervention mean and post-intervention mean or the mean of the experimental (intervention) group and the mean of the control (reference) group [32]. According to Cohen [33] an effect-size of 0.20 is to be considered a small effect, 0.50 a medium effect, and 0.80 a large effect. In order to view the $d$ values in context, Confidence Intervals $(95 \%)$ and Cohen's $U_{3}$ were chosen in the current study. The $U_{3}$ measure indicates the percentage of the participants in the intervention condition who score above the mean for the reference condition.

Typically, in meta-analyses, it is customary to differentiate between random and fixed models [34] in computing Confidence Intervals. In random models, calculations are based on the number of studies included, whereas in fixed models calculations are based on the number of participants. There are advantages and disadvantages with regard to both designs, but Rosenthal and DiMatteo [34] argued, despite reduced power, in favor of a random design, since the results will be more generalizable. This type of argument may also be applied to calculations of Cohen's $d$, that is in the choice between the advantage/disadvantage of basing the results on the number of participants in the studies. According to Rosenthal [29] it may not always be appropriate to weight the results based on the number of participants for example when a single comprehensive study is included. Andersson [31], comparing the difference between large studies and meta-analyses concluded that large studies and metaanalyses frequently yield disparate results, even though the differences between comprehensive studies and metaanalyses are not dramatic. According to Andersson [31] it is not advisable to weight the results on the basis of the number of participants if the $N$ of each study exceeds $N=10$. The present meta-analysis adhered to this recommendation.

Given that the studies included in the present investigation differ in characteristics such as time span and design, it was deemed important to examine such potential differences. Independent Samples $t$-tests showed no significant difference $(p=0.126)$ for Cohen's $d$ regarding studies that lasted for 12 months $(n=5)$, or 24 months or longer $(n=12)$. Further, Independent Samples $t$-tests showed no significant difference concerning Cohen's $d$ in regard to RCT (Randomized Controlled Trial) studies or non-RCT studies for Symptoms ( $p=$ $0.826)$, Functioning $(p=0.931)$, or Well-being $(p=0.176)$. Finally, Independent Samples $t$-tests showed no significant difference $(p=0.766)$ regarding studies with a within-design $(n=8)$, or a between-design $(n=9)$.

In order to maximize homogeneity for statistical synthesis [35], results were segregated by study type (randomized controlled trials and non-randomized observational studies) and outcome parameters (symptoms, functioning and wellbeing). This procedure resulted in six subgroups for the synthesis. Since the current meta-analysis of 17 studies may be regarded as one of medium or small size, Levene's test which does not require normality of the underlying data was chosen. The homogeneity analysis (Levene, $5 \%$ level) 
Table 1. Effect Sizes (Cohen's $d$ ) by Study and Outcome Measure in Regard to Experimental or Intervention Measurements (exp/int), Control Groups (Con), Pre-Intervention or Reference Measurements (ref), within-Subjects Design (with) and Between-Subjects Design (Bet)

\begin{tabular}{|c|c|c|c|c|c|}
\hline Outcome measure & $\mathbf{N}_{\text {exp } / \text { int }}$ & $\mathbf{N}_{\text {con }}$ & $\mathbf{N}_{\text {ref }}$ & $d_{\text {with }}$ & $d_{\text {bet }}$ \\
\hline \multicolumn{6}{|l|}{ Symptoms } \\
\hline Economou, Palli, Peppou, Madianos [16] & 60 & & 60 & 1.02 & \\
\hline EQUIP [17] & 42 & & 42 & 0.14 & \\
\hline Grawe, Falloon, Widen, Skogvoll [18] & 30 & 20 & & & 0.60 \\
\hline Malm, Allebeck, Ivarsson [19] & 12 & & 12 & 0.15 & \\
\hline Malm, Ivarsson, Allebeck, Falloon [20] & 51 & 33 & & & 0.15 \\
\hline Mastroeni, Bellotti, Pellegrini, et al. [21] & 51 & & 46 & & 0.49 \\
\hline Montero, Ascenio, Hernández, et al. [22] & 46 & 41 & & & 0.03 \\
\hline Nordén, Ivarsson, Malm, Norlander [10] & 133 & & 1243 & & 0.48 \\
\hline Pioli, Vittorielli, Gigantesco, et al. [23] & 29 & 26 & & & 0.26 \\
\hline Ryu, Mizuno, Sakuma, et al. [24] & 78 & & 60 & 0,31 & \\
\hline Stewart, Gedye, Fernando [25] & 24 & & 24 & 2.50 & \\
\hline Sungur, Soygür, Güner, et al. [26] & 50 & 50 & & & 1.79 \\
\hline Veltro, Magliano, Morosini, et al. [27] & 12 & 12 & & & 0.61 \\
\hline Veltro, Mazza, Vendittelli, et al. [28] & 12 & & 12 & 0.21 & \\
\hline \multicolumn{6}{|l|}{ Function } \\
\hline Economou, Palli, Peppou, Madianos [16] & 60 & & 60 & 0.41 & \\
\hline EQUIP [17] & 42 & & 42 & 1.73 & \\
\hline Grawe, Falloon, Widen, Skogvoll [18] & 30 & 20 & & & 0.70 \\
\hline Malm, Allebeck, Ivarsson [19] & 12 & & 12 & 0.78 & \\
\hline Malm, Ivarsson, Allebeck, Falloon [20] & 51 & 33 & & & 0.52 \\
\hline Mastroeni, Bellotti, Pellegrini, et al. [21] & 51 & & 46 & & 0.71 \\
\hline Montero, Ascenio, Hernández, et al. [22] & 46 & 41 & & & 0.17 \\
\hline Nordén, Eriksson, Kjellgren, Norlander [11] & 80 & & 80 & 0.47 & \\
\hline Nordén, Ivarsson, Malm, Norlander [10] & 133 & & 1243 & & 0.70 \\
\hline Pioli, Vittorielli, Gigantesco, et al. [23] & 29 & 26 & & & 0.64 \\
\hline Ryu, Mizuno, Sakuma, et al. [24] & 78 & & 60 & 0.65 & \\
\hline Stewart, Gedye, Fernando [25] & 24 & & 24 & 2.36 & \\
\hline Sungur, Soygür, Güner, et al. [26] & 50 & 50 & & & 2.84 \\
\hline Veltro, Magliano, Morosini, et al. [27] & 12 & 12 & & & 0.96 \\
\hline Veltro, Mazza, Vendittelli, et al. [28] & 12 & & 12 & 0.20 & \\
\hline \multicolumn{6}{|l|}{ Well-being } \\
\hline Berglund, Vahlne, Edman [14] & 14 & & 17 & & 1.62 \\
\hline Economou, Palli, Falloon [15] & 51 & & 50 & 0.39 & \\
\hline Economou, Palli, Peppou, Madianos [16] & 60 & & 60 & 0.48 & \\
\hline EQUIP [17] & 42 & & 42 & 0.90 & \\
\hline Grawe, Falloon, Widen, Skogvoll [18] & 30 & 20 & & & 1.44 \\
\hline Malm, Allebeck, Ivarsson [19] & 12 & & 12 & 0.03 & \\
\hline Malm, Ivarsson, Allebeck, Falloon [20] & 51 & 33 & & & 0.55 \\
\hline Montero, Ascenio, Hernández, et al. [22] & 46 & 41 & & & 0.35 \\
\hline
\end{tabular}


Table 1. contd....

\begin{tabular}{|c|c|c|c|c|c|}
\hline Outcome Measure & $\mathbf{N}_{\text {exp/int }}$ & $\mathbf{N}_{\text {con }}$ & $\mathbf{N}_{\text {ref }}$ & $d_{\text {with }}$ & $d_{\text {bet }}$ \\
\hline Nordén, Ivarsson, Malm, Norlander [10] & 133 & & 1243 & & -0.02 \\
\hline Sungur, Soygür, Güner, et al. [26] & 50 & 50 & & & 2.15 \\
\hline Veltro, Magliano, Morosini, et al. [27] & 12 & 12 & & & 1.33 \\
\hline
\end{tabular}

Table 2. Effect Sizes (Cohen's $d$ ), Confidence Intervals (95\%), and Percentage Above Mean (Cohen's $\left.U_{3}\right)$ Regarding Comparisons of Experimental and Control Groups for Studies with a Randomized Control Group Design (RCT)

\begin{tabular}{|c|c|c|c|c|c|}
\hline Outcome Measure & No $_{\text {studies }}$ & No $_{\text {participants }}$ & $\boldsymbol{d}_{\text {exp-control }}$ & CI $_{95}$ & $\boldsymbol{U}_{\mathbf{3}}$ \\
\hline \hline All measures & 6 & 400 & 0.87 & $0.47-1.28$ & 80.78 \\
\hline Symptoms & 6 & 400 & 0.57 & $-0.10-1.25$ & 71.57 \\
\hline Function & 6 & 400 & 0.93 & $-0.10-1.96$ & 82.38 \\
\hline Well-being & 5 & 345 & 1.16 & $0.26-2.07$ & 87.70 \\
\hline
\end{tabular}

Table 3. Effect Sizes (Cohen's $d$ ), Confidence Intervals (95 \%), and Percentage Above Mean (Cohen's $U_{3}$ ) Regarding Comparisons of Intervention and Reference Measurements for Observational Studies (non-RCT)

\begin{tabular}{|c|c|c|c|c|c|}
\hline Outcome Measure & No $_{\text {studies }}$ & No $_{\text {participants }}$ & $\boldsymbol{d}_{\text {int-reference }}$ & CI $_{\mathbf{9 5}} \%$ & $\boldsymbol{U}_{\mathbf{3}}$ \\
\hline \hline All measures & 11 & 1863 & 0.73 & $0.43-1.03$ & 76.73 \\
\hline Symptoms & 8 & 1701 & 0.66 & $0.00-1.33$ & 74.54 \\
\hline Function & 9 & 1781 & 0.89 & $0.35-1.43$ & 81.33 \\
\hline Well-being & 6 & 1572 & 0.57 & $-0.06-1.21$ \\
\hline
\end{tabular}

regarding Cohen's $d$ yielded no significant effects for randomized or non-randomized conditions [Levene $(1,38)=$ $0.61, p=0.440]$ or for outcome parameters $[$ Levene $(2,37)=$ $0.16, p=0.854]$ or as expected for the six subgroups ( $p s>$ $0.05)$ thereby indicating homogeneity.

\section{RESULTS}

\section{Description of Studies}

An overview of the studies included in the current metaanalysis is given in Table $\mathbf{1}$, including the number of participants in the different conditions by study and outcome are (a) experimental or intervention measurements, (b) control groups, (c) pre-intervention or reference measurements. Cohen's $d s$ regarding within-subjects design studies and between-subjects design studies are also given. As mentioned above, six of the studies were characterized by an RCT design and all of the participating clients were diagnosed as having schizophrenic disorders. The quality of those studies was checked in accordance with the system of GRADE (Grades of Recommendations Assessment, Development and Evaluation), frequently used to rate the power of evidence and appropriateness for implementation [36]. Grading was done on 4-point scales, Categories of Qualities of Evidence $(C Q E)$, where 1 indicated very low quality, 2 for low quality, 3 for moderate quality, and 4 for high quality. In the assessments, attention was also paid to five determinants of quality: detailed design and execution, inconsistency, indirectness, reporting bias, and imprecision. The assessments were carried out by two independent judges, and the averaged results indicated good levels of quality for Symptoms (CQE
=3.75), Functioning $(C Q E=3.83)$, and Well-being $(C Q E=$ 3.60) which yielded a mean $C Q E$ score of 3.73 .

The eleven observational studies exhibited great variation in terms of sample size. The median number of participants for those studies was 55, but two of the studies had only 12 participants, and one study had as many as 1376 participants. The large study [10] consisted of three parts each lasting 24 months, conducted at nine participating centers. Since there were no statistical differences on either symptoms or functioning the three parts were analyzed together in order to make comparisons possible between clients who received standard care $(n=1243)$ and those who were treated in accordance with the RACT method $(n=133)$. Given that the large study accounted for $75 \%$ of all the participants and 28 $\%$ of the measures of intervention in the non-RCT condition, it was necessary in the current meta-analysis to analyze the specific results of this unique study. It was done simply by redoing the analysis with this particular study removed, i.e. with the remaining 10 studies $(n=487)$ from the non-RCT condition. The results of the analysis indicated that the Cohen's $d$ increased slightly for all outcome variables, and the effect size for "All Measures" increased to $d=0.78$.

\section{Outcomes}

Table 2 shows an overall effect size of 0.87 for studies with a randomized control group design (RCT), considered a large effect by Cohen [33]. Large effects were also noted for Functioning and Well-being, whereas Symptoms yielded a medium effect. Table 3 shows an overall effect size of 0.73 for observational studies with pre-post or interventionreference comparisons (non-RCT), a result which is slightly 
below the benchmark for a large effect. Functioning landed clearly above the limit, whereas Symptoms and Well-being landed at a medium level.

In thirteen of the studies, all of the clients had diagnoses within the psychotic spectrum, whereas four of the non-RCT studies $[10,11,21,25]$ also included a minority of clients with different psychiatric diagnoses $(17 \%, 23 \%, 24 \%, 35 \%)$ in a total of 308 individuals. No information was evident suggesting that non-psychotic clients would benefit less from the use of Resource Groups. On the contrary, the four studies contained material that suggested that non-psychotic clients also benefited from the RACT method. The largest study in the current meta-analysis [10] showed that clients treated with RACT obtained better results in terms of symptoms (GAFSymptoms) and functioning (GAF-Function) compared with those who received standard care. A follow-up statistical analysis showed that the improvements were characteristic both of clients with diagnoses within the psychotic spectrum and of clients with non-psychotic diagnoses.

\section{The File-Drawer Problem}

In a meta-analysis it is advisable to take into account "the File-drawer problem" [37], a phenomenon emphasizing the risk for non-significant studies being left unpublished (i e, they end up in the "drawer"). If that is the case, the metaanalysis becomes distorted. Rosenthal [38] developed formulas to calculate the so-called fail-safe $N$, i.e. a measure of how many studies must remain unpublished in the drawer, studies which also go against the direction of the hypothesis, in order for the author thus to draw erroneous conclusions, when indeed the results of the meta-analysis in reality do not show a true effect.. If the critical number of unpublished studies is less than the fail-safe $N$, there is no file-drawer problem. The fail-safe $N$ for the current meta-analysis was calculated [38] and indicated no file-drawer problem (failsafe $N=95$ ). The authors of the current study have utilized a great amount of knowledge of the state of research from the international OTP project as well as from clinical activities with the RACT model and would therefore like to make the point that it is highly unlikely that there exist 95 unpublished articles with findings contradicting the results of the current meta-analysis.

\section{DISCUSSION}

The current investigation had two hypotheses: (a) the treatment of clients with Resource group Assertive Community Treatment (RACT) will have positive effects for psychotic clients, and (b) the treatment of clients with Resource group Assertive Community Treatment (RACT) may be of use to clients within the entire psychiatric spectrum. Considering the first hypothesis, the results were completely in line with those of earlier reports $[12,13]$, suggesting that the method now labeled Resource group Assertive Community Treatment (RACT) is a successful method for the treatment of psychotic clients. The similar results between studies may indicate that the resource group, with its person-centered methodology, has proven to be adaptable to different welfare systems and cultures. All of the studies using a randomized control group design (RCT) consisted exclusively of clients with psychotic diagnoses, and the same fact was true of seven of the observational studies. Only four of the observa- tional studies included clients with non-psychotic diagnoses, and within those studies the clients with non-psychotic diagnoses constituted a minority. Despite this fact, the results indicated that RACT may, as suggested by the second hypothesis, benefit clients with non-psychotic diagnoses, as well. Naturally, this notion must be replicated in additional studies. Nevertheless, even now one might suggest to interested clinics that working with Resource Groups may be worthwhile even for a more comprehensive group of clients.

The current meta-analysis comprised three outcome variables: Symptoms, Functioning, and Well-being. A medium effect was noted for Symptoms for both RCT and nonRCT studies, whereas analyses of Functioning yielded large effects for both types of design. Symptomatic remission criteria have been developed for schizophrenia $[39,40]$, and at the same time research on remission has increasingly paid more attention to the need for a concept of functional remission. Our work with Resource Groups primarily focuses on functioning [11], since experience indicates that remission is more easily attained. If the client learns to deal with different situations with the aid of good strategies, remission may be attained even when several symptoms remain. Better functioning will yield a better platform for recovery.

It is more difficult to explain why the RCT studies noted a large effect for Well-being, while the non-RCT studies evinced a medium effect. One explanation might however be that even at the planning and inception of the studies included in the current meta-analysis it was obvious that wellknown measurement instruments for symptoms and functioning should be employed. In contrast, there was a greater variation regarding measures of well-being. It is worth noting that within the RCT condition, all relevant studies included except one, assessments for well-being, whereas in the non-RCT studies almost half were lacking in such assessments. Despite these weaknesses, there exists a total effect showing that work with Resource Groups contributes to client well-being.

The current investigation contains an array of analyses delineating various characteristics of the studies included, in which it was evident that there were no significant differences regarding Cohen's $d$ among studies (a) lasting less than a year or two years and more, (b) had a RCT (Randomized Controlled Trial) design, or consisted of observational studies, (c) or had a within-design or between-design. Furthermore, a homogeneity analysis yielded no significant effects for randomized or non-randomized conditions or for outcome parameters suggesting homogeneity. All of those analyses give us the courage to compile results from the RCT and the non-RCT studies into one single "grand total measure" for all the 17 studies included, thereby providing a large total effect size $\left(d=0.80, U_{3}=78.81\right)$. The results of the current meta-analysis would benefit from a comparison with those of other meta-analyses with comparable treatment programs. There now exists a large number of meta-analyses addressing various treatment methods and client groups. Furthermore, one may now find meta-analyses of meta-analyses (i. e., meta-meta-analyses). In one such classic meta-metaanalysis based on 302 meta-analyses of psycho-social interventions, Lipsey and Wilson [41] found a mean effect size of $d=0.50$. Mojtabai, Nicholson, and Carpenter [42] conducted 
a meta-analysis based on 106 studies of clients with the diagnosis of schizophrenia. The treatment effect for psychosocial therapies in combination with medication was compared with control groups where the clients received medication only, and there was an overarching effect size of $d=$ 0.39 in favor of the combined treatment. There is a rich flora of meta-analyses geared exclusively toward Assertive Community Treatment. However, we have not found any metameta-analyses of those meta-analyses, but browsing them suggests that the most common effect sizes appear to be small to medium according to the criteria advocated by Cohen [33]. This finding is consistent with the results of studies of many new social programs and treatments. In a review [43] of two meta-meta-analyses (actually a metameta-meta-analysis!), one of the analyses based on 24 metaanalyses and the other based on 36 meta-analyses, the conclusion was drawn that a consistent finding from various meta-analyses is that meta-analyses of most new treatment programs yield at best small to moderate effects.

\section{CONCLUSION}

The current meta-analysis is the first meta-analysis conducted within the tradition of Assertive Community Treatment and intensive case management using Resource Groups. RACT is distinctive in that the ACT team consists not only of professionals but also of the client and his/her significant others. In addition, the treatment originates with the needs and wishes of the client. This type of approach strengthens the self-confidence and competence of the client. The empowerment of the client, in turn, appears to be the major driving force for progress in treatment [11]. The conclusions of the meta-analysis were that the treatment of clients with Resource Group Assertive Community Treatment yields positive effects for clients with psychoses and that the method may be of use for clients within the entire psychiatric spectrum. Against this background we would like to formulate a hypothesis for future studies: The mere existence of Resource Groups helps this particular type of Assertive Community Treatment produce large effect sizes.

\section{CONFLICT OF INTEREST}

The authors confirm that this article content has no conflicts of interest.

\section{ACKNOWLEDGEMENTS}

This study followed the ethical standards of the World Medical Association declaration of Helsinki concerning Ethical Principles of Medical Research Involving Human Subjects.

\section{REFERENCES}

[1] Simmons S, Coid J, Joseph P, Marriott S, Tyrer P. Community mental health team management in severe mental illness: a systematic review. Br J Psychiatry 2001; 178: 497-502

[2] van Veldhuizen JR. FACT: A Dutch version of ACT. Community Ment Health J 2007; 43: 421-33.

[3] Stein LL. A historical review of the Madison model of community care assertive community treatment. Hosp Community Psychiatry 1990; 41: 649-51.

[4] Lewin Group. Assertive Community Treatment literature review. Prepared for health care and financing administration and substance abuse and mental health services administration. Falls Church, VA: The Lewin Group 2000.
[5] Falloon IRH. Family management of schizophrenia. Baltimore: John Hopkins University Press 1985.

[6] Falloon IRH and the Optimal Treatment Project Collaborators. Optimal treatment for psychosis in an international multisite demonstration project. Psychiatr Serv 1999; 50: 615-8.

[7] Falloon IHR, Coverdale JH, Brooker C. Psychosocial interventions in schizophrenia: a review. Int J Ment Health 1996; 25: 3-21.

[8] Falloon IRH, Laporta M, Fadden G, Graham-Hole V. Managing stress in families: Cognitive and behavioral strategies for enhancing coping skills. London: Routledge 1993.

[9] Malm U. Case management. Evidenbaserad integrerad psykiatri [Case management. Evidenced based integrative psychiatry]. Lund, Sweden: Studentlitteratur 2002.

[10] Nordén T, Ivarsson B, Malm U, Norlander T. Gender and treatment comparisons in a cohort of patients with psychiatric diagnoses. Soc Behav Pers 2011; 39: 1073-86.

[11] Nordén T, Eriksson A, Kjellgren A, Norlander T. Involving clients and their relatives and friends in the psychiatric care. Case managers' experiences of training in Resource group Assertive Community Treatment. Psych J 2012; 1: 15-27.

[12] Falloon IRH, Montero I, Sungur M, et al. The Otp Collaborative Group: Implementation of evidence-based treatment for schizophrenic disorders: two-year outcome of an international field trial of optimal treatment. World Psychiatry 2004; 3: 104-9.

[13] Hayman-White K, Happell B. Critique of falloon and the optimal treatment project. Int J Ment Health Nurs 2007; 16: 44-9.

[14] Berglund N, Vahlne JO, Edman Å. Family intervention in schizophrenia. Impact on family burden and attitude. Soc Psychiatry Psychiatr Epidemiol 2003; 38: 116-21.

[15] Economou M, Palli A, Falloon IRH. Violence, misconduct and schizophrenia: Outcome after four years of optimal treatment. Clin Pract Epidemiol Ment Health 2005; 1: 3.

[16] Economou M, Palli A, Peppou L, Madianos M. Recovery from Schizophrenia: a four-year study of an inner city cohort. Community Ment Health J 2011; 47: 660-7.

[17] EQUIP. Community and residential support outcome report. Report on EQUIP client, family and service outcomes: Auckland and Waitemata Districts. Auckland, New Zealand: Windsor Park Baptist Church 2007.

[18] Grawe RW, Falloon IRH, Widen JH, Skogvoll E. Two years of continued early treatment for recent-onset schizophrenia: a randomized controlled study. Acta Psychiatr Scand 2006; 114: 328-36.

[19] Malm U, Allebeck P, Ivarsson B. The effectiveness of integrated care: a naturalistic study. Conference of Integrative and Flexible Assertive Community Treatment; 2011 Nov 18; Göteborg, Sweden: Jonsered Herrgård 2012.

[20] Malm U, Ivarsson B, Allebeck P, Falloon IRH. Integrated care in schizophrenia: a 2-year randomized controlled study of two community-based treatment programs. Acta Psychiatr Scand 2003; 107: 415-23.

[21] Mastroeni A, Bellotti C, Pellegrini E, Galletti F, Lai E, Falloon IRH. Clinical and social outcomes five years after closing a mental hospital: a trial of cognitive behavioral interventions. Clin Pract Epidemiol Ment Health 2005; 1: 25.

[22] Montero I, Ascenio A, Hernández I, et al. Two strategies for family intervention in schizophrenia: A randomized trial in a Mediterranean environment. Schizophr Bull 2001; 27: 661.

[23] Pioli R, Vittorielli M, Gigantesco A, et al. Clin Pract Epidemiol Ment Health 2006; 2: April 03. Available from: http://www.cpementalhealth.com/content/2/1/5670.

[24] Ryu Y, Mizuno M, Sakuma K, et al. Deinstitutionalization of longstay patients with schizophrenia: the 2-year social and clinical outcome of a comprehensive intervention program in Japan. Aust N Z J Psychiatry 2006; 40: 462-70.

[25] Stewart MW, Gedye R, Fernando A. Hearing their voices: A multidimensional evaluation of an early intervention for psychosis team In Turner B, Nightingale S, Mulder R, Maginness A, Eds. Evaluation of early intervention for psychosis services in New Zealand: What works? Auckland, New Zealand: Health Research Council of New Zealand; 2002; pp. 179-91.

[26] Sungur M, Soygür H, Güner P, Üstün B, Cetin I, Falloon IHR. Identifying an optimal treatment for schizophrenia: A 2-year randomized controlled trial comparing integrated care to a high-quality routine treatment. Int J Psychiatry Clin Pract 2011; 15: 118-27. 
[27] Veltro F, Magliano L, Morosini P, et al. Studio controllato randomizzato di un intervento psicoeducativo familiare. Epidemiol Psichiatr Soc 2006; 15: 44-51.

[28] Veltro F, Mazza M, Vendittelli N, Alberti M, Casacchia M, Roncone R. A comparison of effectiveness of problem solving training and of cognitive-emotional rehabilitation on neurocognition, social cognition and social functioning in people with schizophrenia. Clin Pract Epidemiol Ment Health 2011; 7: 123-32.

[29] Rosenthal R. Meta-analytic procedures for social research. Newbury Park, CA: Sage 1991.

[30] Wästlund E, Norlander T, Archer T. Exploring cross-cultural differences in self-concept: A Meta-analysis of SDQ1. Cross Cult Res 2001; 35: 280-302.

[31] Andersson G. Metaanalys. Metoder, tillämpningar och kontroverser Meta-analysis. Methods, applications and controversies. Lund, Sweden: Studentlitteratur 2003.

[32] van Dierendonck D, te Nijenhuis J. Flotation restricted environmental stimulation therapy (REST) as a stress-management tool: a meta-analysis. Psychol Health 2005; 20: 405-12.

[33] Cohen J. Statistical power analysis for the behavioral sciences. Hillsdale, NJ: Lawrence Erlbaum 1988.

[34] Rosenthal R, DiMatteo MR. Meta-analysis: recent developments in quantitative methods for literature reviews. Annu Rev Psychol 2001; 52: 59-82.
[35] Petitti DB. Meta-analysis, decision analysis, and cost-effectiveness analysis: Methods for quantitative synthesis in medicine. NY: Oxford University Press 2000.

[36] Schünemann, H. GRADE (Grades of Recommendations Assessment, Development and Evaluation 2008. Available 2012-02-18 from http://www.igh.org/Cochrane/GRADE/schunemannGRADEmay2008.pdf

[37] Rosenthal R. The "file drawer problem" and tolerance for null results. Psychol Bull 1979; 86: 638-41.

[38] Rosenthal R. Meta-analysis: a review. Psychosom Med 1991; 53: 247-71.

[39] Harvey PD, Helldin L, Bowie CR, et al. Performance-based measurement of functional disability in schizophrenia: A cross-national study in the United States and Sweden. Am J Psychiatry 2009; 166: 821-7.

[40] Helldin L, Kane JM, Hjärthag F, Norlander T. The importance of cross-sectional remission in schizophrenia for long-term outcome, a clinical prospective study. Schizophr Res 2009; 115: 67-73.

[41] Lipsey MW, Wilson DB. The efficacy of psychological, educational, and behavioral treatment. Confirmation from meta-analysis. Am Psychol 1993; 48: 1181-209.

[42] Mojtabai R, Nicholson RA, Carpenter BN. Role of psychosocial treatments in management of schizophrenia: a meta-analytic review of controlled outcome studies. Schizophr Res 1998; 24: 569-87.

[43] Cook TD, Cooper H, Cordray DS, et al. Meta-Analysis for Explanation: A Casebook. NY: Russell Sage Foundation 1992.

Received: March 2, 2012

(C) Nordén et al.; Licensee Bentham Open.

This is an open access article licensed under the terms of the Creative Commons Attribution Non-Commercial License (http://creativecommons.org/licenses/by-nc/3.0/) which permits unrestricted, non-commercial use, distribution and reproduction in any medium, provided the work is properly cited. 